\title{
Determination of Landau free-energy parameters by dielectric measurements in the ferroelectric TSCC
}

\section{Authors: J. Bornarel \& V. Hugo Schmidt}

This is an author-created, un-copyedited version of an article published in Journal of Physics C: Solid State Physics. IOP Publishing Ltd is not responsible for any errors or omissions in this version of the manuscript or any version derived from it. The Version of Record is available online at http://dx.doi.org/10.1088/0022-3719/14/14/012.

J. Bornarel and V.H. Schmidt, "Determination of Landau free-energy parameters by dielectric measurements in the ferroelectric TSCC," Journal of Physics C: Solid State Physics 14, 2017-2025 (1981). http://dx.doi.org/10.1088/0022-3719/14/14/012

Made available through Montana State University's $\underline{\text { ScholarWorks }}$ scholarworks. montana.edu 


\title{
Determination of Landau free-energy parameters by dielectric measurements in the ferroelectric $\mathrm{TSCC}$
}

\author{
$\mathrm{J}$ Bornarel and V H Schmidt $\dagger$ \\ Université Scientifique et Médicale de Grenoble, Laboratoire de Spectrométrie Physique \\ (associé au CNRS), Boîte Postale no 53X 38041 Grenoble Cedex, France
}

Received 15 August 1980

\begin{abstract}
We measured the adiabatic dielectric permittivity $\varepsilon_{b}$ of tris-sarcosine calcium chloride (TSCC) at $400 \mathrm{~Hz}$ near the ferroelectric transition for DC bias fields up to $5000 \mathrm{~V} \mathrm{~cm}^{-1}$. We found $\varepsilon_{\infty}=4.2 \pm 0.2$, Curie-Weiss constant $C^{+}=38.3 \pm 0.3 \mathrm{~K}, T_{0}=$ $129.4 \pm 0.1 \mathrm{~K}$, and Landau coefficients $A=(2.95 \pm 0.2) \times 10^{9} \mathrm{~V} \mathrm{~m} \mathrm{~A}^{-1} \mathrm{~s}^{-1} \mathrm{~K}^{-1}$ and $B=(3.5 \pm 0.1) \times 10^{15} \mathrm{~V} \mathrm{~m}^{5} \mathrm{~A}^{-3} \mathrm{~s}^{-3}$. We observed evidence of an internal bias field as reported previously by others.
\end{abstract}

\section{Introduction}

Tris-sarcosine calcium chloride (TSCC) has a paraelectric (PE) to ferroelectric (FE) transition near $130 \mathrm{~K}$. Its structure was found (Ashida et al 1972) to be orthorhombic Pnma in the PE phase, with $Z=4$ formula units of $\left(\mathrm{CH}_{3} \mathrm{NHCH}_{2} \mathrm{COOH}\right)_{3} \mathrm{CaCl}_{2}$ per unit cell. The FE phase is presumed (Makita 1965) to remain orthorhombic, with space group $\operatorname{Pn} 2_{1} a$ and $Z=4$. Dielectric investigations of TSCC have already been made by several groups. Such studies at high pressure have partly elucidated the phase diagram. The PE-FE transition temperature $T_{\mathrm{c}}$ increases rapidly with pressure (Windsch and Schmidt 1979, 1980). At higher pressure a disappearance (Hegenbarth and Schmidt 1980, Fujimoto et al 1980) of the dielectric anomaly was noted. This disappearance was found (Schmidt 1980) to coincide with a transition to a third, probably antiferroelectric (AFE), phase which exists only at high pressure, the common point for the three phases being at $5.03 \mathrm{kbar}$ and $176.8 \mathrm{~K}$. The most extensive dielectric measurements have been made at ambient pressure. The effects of temperature and DC electric field were studied (Makita 1965, Levstik et al 1975, Sorge and Straube 1979). The latter authors investigated also the effect of the amplitude and frequency of the measuring field and reported unusual results for the effect of a DC field $E$ on the dielectric permittivity $\varepsilon_{b}$ : 'For $E$ smaller than $0.5 \mathrm{kV} \mathrm{cm}^{-1}$ the maximum of $\varepsilon_{b}$ is shifted to lower temperatures, whereas for $E$ greater than $0.5 \mathrm{kV} \mathrm{cm}^{-1}$ it is shifted to higher temperatures with $\mathrm{d} T_{\mathrm{d}} / \mathrm{d} E=0.18$ $\pm 0.02 \mathrm{~K} \mathrm{~cm} \mathrm{kV}^{-1}$. The shift at low field also depends on the sign of $E^{\prime}$.

The purpose of the present work was:

(i) to clarify if possible this behaviour of $\varepsilon_{b}(E, T)$;

$\dagger$ Permanent address: Department of Physics, Montana State University, Bozeman, Montana 59717, USA. 
(ii) to determine precisely the coefficients of the Landau free-energy expansion from dielectric measurements.

\section{Experimental}

The first sample was taken from a crystal grown by us by the evaporation method from aqueous solution of $98 \%$ pure sarcosine and calcium chloride. The second and third samples were from recrystallised TSCC crystals and presumably have higher purity. TSCC crystals generally grow with ferroelastic twins (domains) (Sawada et al 1977) which are observable under a polarising microscope for light propagating along the $a$ axis. Monodomain portions were cleaved from these crystals and replaced in solution, after which they generally remained monodomain during further growth. Our samples were made from such ferroelastically monodomain crystals by polishing them with wet filter paper to parallelepiped shape with faces perpendicular to crystallographic axes. Dimensions were $a=3.25 \mathrm{~mm}, b=2.15 \mathrm{~mm}, c=5.95 \mathrm{~mm}$ for the third sample, for which the quantitative results reported herein were measured. Gold electrodes were deposited by evaporation on the faces perpendicular to the FE $b$ axis. Electrical contacts to the samples were made by two methods.

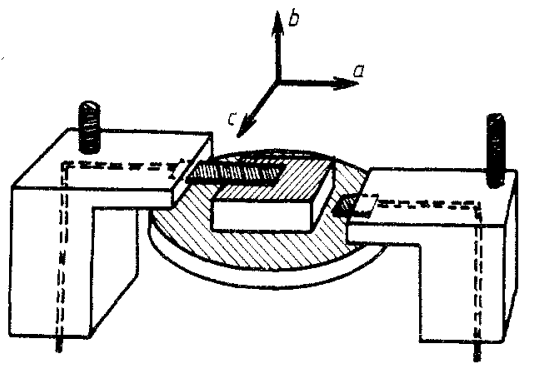

(a)

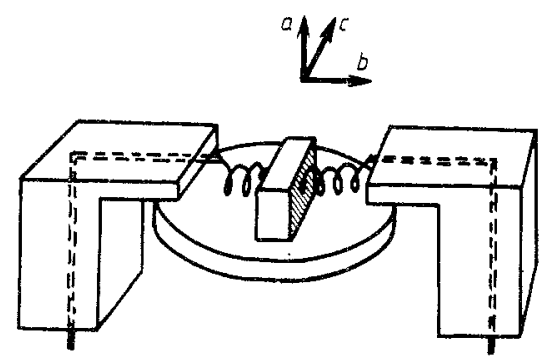

(b)

Figure 1. (a) First method for providing electrical contacts to crystal. (b) Second method for providing electrical contacts to crystal.

In the first method, used with the first two samples and shown in figure $1(a)$, the sample was placed on a metallised support. Its upper face was contacted by a copper foil attached to a Teflon block whose position was adjustable to give the desired contact pressure. Spots of silver paste assured good contact of the crystal electrodes to the support plate and the copper foil. A disadvantage of this method is that differential thermal expansion may cause excessive compression along $b$ at low temperature.

In the second method, illustrated in figure $1(b)$ and employed for the third sample, a face perpendicular to $a$ rested on an insulating block. Contacts were made by very weak beryllium-copper coil springs attached to the $b$ faces by drops of silver paint, thus achieving a very weak and nearly temperature-independent compressive force along $b$.

The samples were placed in a cell designed for electrical measurements and optical viewing. Temperature was regulated by a heater located on the thermal path between the crystal and a liquid nitrogen reservoir. Helium at low pressure acted as an exchange gas to maintain essentially uniform sample temperature despite radiation losses through 
the viewing window. A Eurotherm type 040 temperature regulator provided stability of $10^{-2} \mathrm{~K}$ or better, as measured with a Rosemount platinum resistor and a Dana 5000 digital multimeter readout. The relative precision of two measurements near $T_{\mathrm{c}}$ was of order $0.01 \mathrm{~K}$, but the absolute accuracy was only several tenths of a degree.

The dielectric measurements were performed at $400 \mathrm{~Hz}$ and $1 \mathrm{~V}$ RMS with a General Radio type 1673 automatic capacitance bridge. The precision for $C$ was $0.05 \mathrm{pF}$, and for $\tan \delta$ varied from 0.05 for low values of $C$ near room temperature to 0.001 near the transition. An appropriate circuit permitted application of DC voltages up to $\pm 1000 \mathrm{~V}$ to the crystal while isolating the measuring bridge from this DC voltage.

The experimental procedure consisted in measuring the dielectric permittivity $\varepsilon_{b}(E, T)$ at a fixed temperature $T$ for all of the desired bias-field values $E$, then repeating the process at other temperatures near $T_{c}$. The temperature was regulated for several hours at each new value before beginning dielectric measurements, to ensure thermal homogeneity of the sample. Then $\varepsilon_{b}$ (at $400 \mathrm{~Hz}$ and $1 \mathrm{~V} \mathrm{RMS}$ ) was monitored after each stepwise change in $E$ until it had remained constant for several minutes, at which time its value was recorded. This procedure eliminated possible errors resulting from transient adiabatic temperature changes of the sample caused by changes in $E$, changes which would not be noted by the resistance thermometer which was nearby but did not touch the sample. In fact, however, the new values of $C$ and $\tan \delta$ generally stabilised within a few seconds after the step change in $E$.

\section{Results}

The most readily apparent result is the asymmetry relative to applied field of the real part $\varepsilon_{b}^{\prime}(E)$, of the permittivity. All three samples exhibited at fixed $T \simeq T_{\mathrm{c}}$ a sharp peak of $\varepsilon_{b}^{\prime}(E)$ displaced from $E=0$. In addition, the first two samples exhibited a rounded side peak of $\varepsilon_{b}^{\prime}(E)$ for one polarity only of $E$. Both of these samples were mounted as shown in figure 1(a); one was from a crystal grown from the original solution while the other was from a recrystallised crystal. Accordingly, non-uniform compressive stress rather than crystal perfection seems to govern this asymmetric behaviour.

The third sample whose mounting system shown in figure $1(b)$ caused weaker forces, exhibited no side peak; its $\varepsilon_{b}^{\prime}(E)$ form was symmetric with respect to a positive field value which gave maximum $\varepsilon_{b}^{\prime}$. Such curves for five temperatures near $T_{c}$ are shown in figure 2 ; the highest value of $\varepsilon_{b}^{\prime}$ (more than 1700) is obtained for a temperature of $130.54 \mathrm{~K}$ and a field $E_{\mathrm{m}}$ of $45 \pm 5 \mathrm{~V} \mathrm{~cm}^{-1}$, no effect of $T$ on the field $E_{\mathrm{m}}$ giving maximum $\varepsilon_{b}^{\prime}$ is really noticeable.

The results can also be plotted in the form $\varepsilon_{b}^{\prime}(T)$ for various bias fields as shown in figure 3 . First we note that the temperature $T_{\mathrm{m}}(E)$ giving maximum $\varepsilon_{\mathrm{m}}$ decreases up to $E \simeq E_{\text {bias }}=45 \pm 5 \mathrm{~V} \mathrm{~cm}^{-1}$ and then increases monotonically for larger $E$. A similar effect was observed previously (Sorge and Straube 1979) as noted in the Introduction, except that the internal bias field apparently was about 11 times larger than ours. Although $T_{\mathrm{m}}(E)$ is expected to be a non-linear function of $E$ as explained in the Appendix, we can note that $T_{\mathrm{m}}$ increases by $0.61 \pm 0.06 \mathrm{~K}$ as $E$ increases from 0.14 to $4.65 \mathrm{kV} \mathrm{cm}^{-1}$, which is comparable with the result $\mathrm{d} T_{\mathrm{m}} / \mathrm{d} E=0.18 \pm 0.02 \mathrm{~K} \mathrm{~cm} \mathrm{kV}^{-1}$ quoted previously (Sorge and Straube 1979).

Finally we note that the peak value of $\varepsilon_{b}^{\prime}$ first increases with $E$ and is largest for the $46.5 \mathrm{~V} \mathrm{~cm}^{-1}$ curve, after which it decreases monotonically with increasing $E$. The peak becomes broad and ill-defined at the highest fields used. 


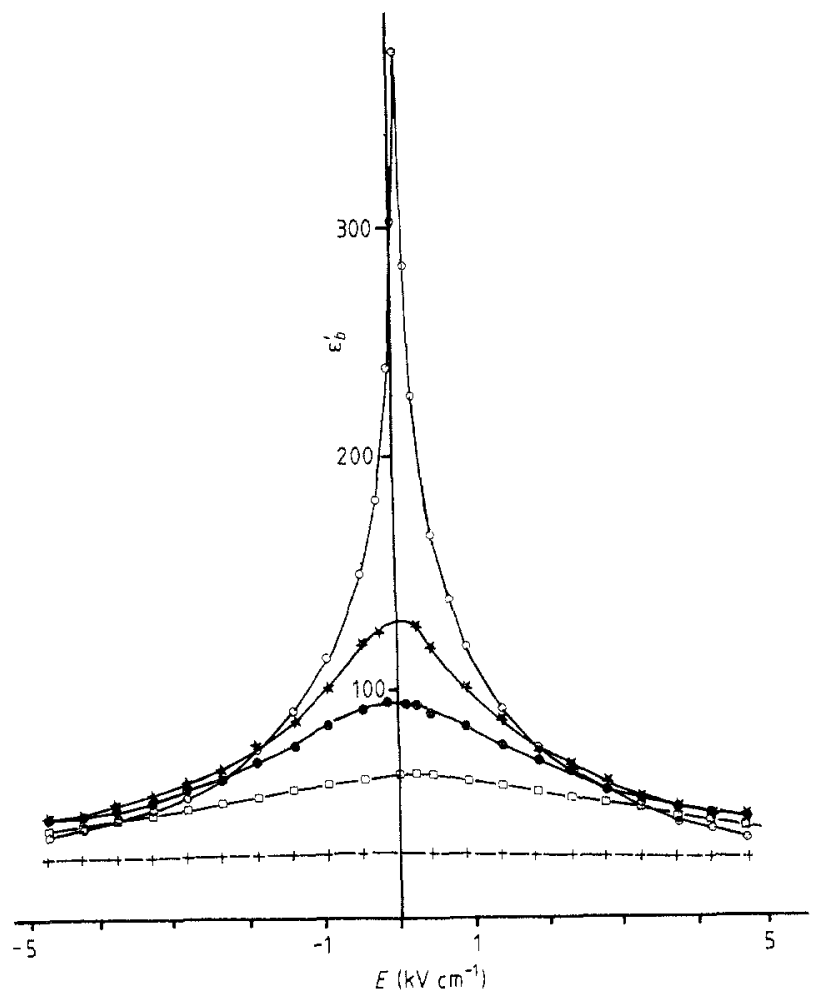

Fìgure 2. $\mathrm{AC}$ (adiabatic) permittivity $\varepsilon_{b}^{\prime}$ versus applied DC field $E$ at five temperatures ; $(\mathrm{O})=130.764 \pm 0.005 \mathrm{~K},(\mathbf{O})=130.94 \pm 0.01 \mathrm{~K},(\mathbf{O})=131.07 \pm 0.01 \mathrm{~K},(\square)=131.33 \pm$ $0.01 \mathrm{~K},(+)=132.055 \pm 0.002 \mathrm{~K}$. Note displacement caused by effective internal bias field of $-45 \pm 5 \mathrm{~V} \mathrm{~cm}^{-1}$.

\section{Discussion}

We analyse our results in terms of the Landau-Devonshire free energy

$$
F=\frac{1}{2} A_{0} t P^{2}+\frac{1}{2 n} B_{2 n} P^{2 n}-E P
$$

in which $P$ is the polarisation, $t=T-T_{0}$ with $T_{0}$ being the Curie-Weiss temperature which is also $T_{\mathrm{c}}$ for non-first-order transitions as studied here, and $A_{0}$ and $B_{2 n}$ are positive constants. If $n=2$ we have a second-order transition while if $n=3$ the transition is at a tricritical point.

From the development based on equation (1) given in the Appendix it is easy to see that the variations of $1 / \varepsilon_{\mathrm{m}}^{\prime}$ and $t_{\mathrm{m}}$ with field $E$ allow determination of $n$ and $B_{2 n}$ if only one $P^{2 n}$ term plays a preponderant role as assumed in equation (1).

We accordingly made the logarithmic plot of $1 / \varepsilon_{\mathrm{m}}^{\prime}$ versus $E$ shown in figure 4 . Between 1 and $5 \mathrm{kV} \mathrm{cm}^{-1}$ the slope is $2 / 3$ as predicted for $n=2$, so that no terms of order higher than $P^{4}$ in the free energy are necessary in the range of $E$ and $T$ covered in this experiment. 


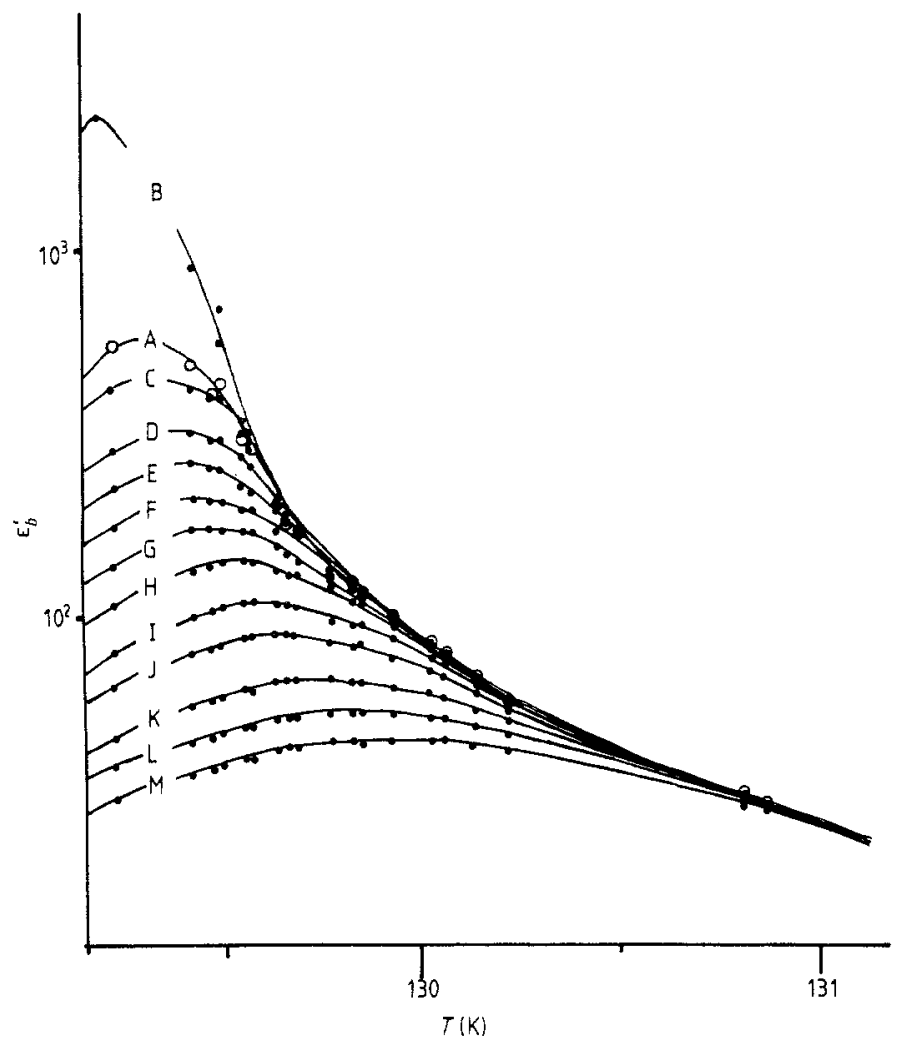

Figure 3. AC (adiabatic) permittivity $\varepsilon_{b}^{\prime}$ versus temperature $T$ for thirteen applied DC fields having magnitudes in $\mathrm{V} \mathrm{cm}^{-1}$ of 0 (A), 46.5 (B), 139.5 (C), 232.6 (D), 325.6 (E), $465(\mathrm{~F})$, $651.2(\mathrm{G}), 930.2(\mathrm{H}), 1395(\mathrm{I}), 1860(\mathrm{~J}), 2791(\mathrm{~K}), 3721$ (L), 4651 (M). Note that highest peak occurs for curve $B$ for which the applied field apparently cancels the internal bias field.

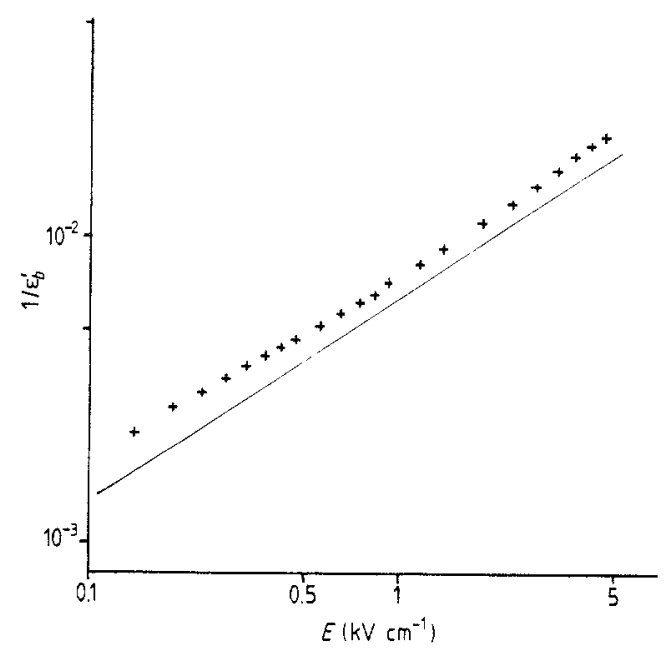

Figure 4. Logarithmic plot of inverse of peak adiabatic permittivity $\left(\varepsilon_{\mathrm{m}}^{\prime}\right)^{-1}$ versus applied field $E$, with line of slope $2 / 3$ for comparison. 
It remains to determine $A_{0} T_{0}$, and $B_{4}$ (henceforth called $B$ ) in equation (1). To find $A_{0}$ and $T_{0}$ we use the law

$$
\varepsilon_{b}^{\prime}=\varepsilon_{\infty}^{\prime}+C^{+} /\left(T-T_{0}\right)
$$

in the form $A_{0}\left(T-T_{0}\right)=\left(\varepsilon_{b}^{\prime}-\varepsilon_{\infty}^{\prime}\right)^{-1}$ where $C^{+}=\left(A_{0} \varepsilon_{0}\right)^{-1}$. Then a plot of $\left(\varepsilon_{b}^{\prime}-\varepsilon_{\infty}^{\prime}\right)^{-1}$ versus $T$ yields $A_{0}$ as the slope and $T_{0}$ as the intercept. We find $\varepsilon_{\infty}^{\prime}=4.2 \pm 0.2, C^{+}=$ $38.3 \mathrm{~K}, A_{0}=(2.95 \pm 0.2) \times 10^{9} \mathrm{~V} \mathrm{~m} \mathrm{~A}^{-1} \mathrm{~s}^{-1} \mathrm{~K}^{-1}$ and $T_{0}=129.4 \pm 0.1 \mathrm{~K}$. To find $B$ we plotted $\left(1 / \varepsilon_{\mathrm{m}}^{\prime}\right)^{3 / 2}$ as a function of $E$ in figure 5 , for which the slope is proportional to $B^{1 / 2}$ as shown in the Appendix. After subtracting the adiabatic correction $B^{\prime}-B$, we found $B=(3.5 \pm 0.1) \times 10^{15} \mathrm{Vm}^{5} \mathrm{~A}^{-3} \mathrm{~s}^{-3}$.

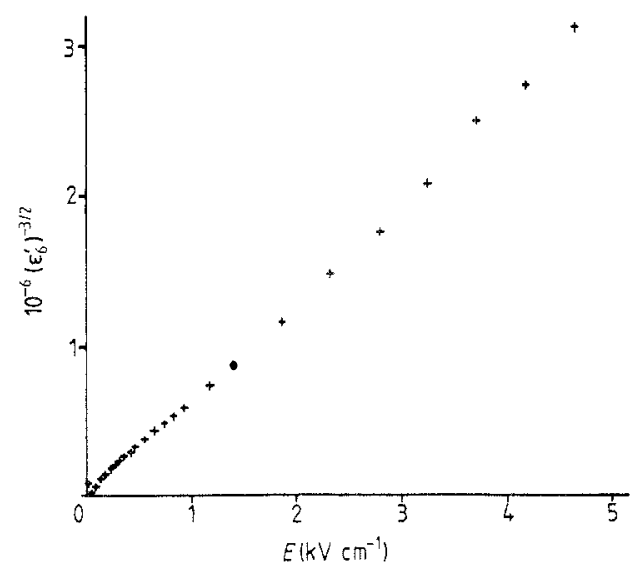

Figure 5. Plot of $-3 / 2$ power of peak adiabatic permittivity, $\left(\varepsilon_{\mathrm{m}}^{\prime}\right)^{-3 / 2}$, versus applied field $E$, having slope proportional to Landau coefficient $B$ plus an adiabatic correction term $\left(B^{\prime}-\right.$ $B)$.

The other law developed in the Appendix, giving $t_{\mathrm{m}}=T_{\mathrm{m}}-T_{0}$ as a function of $E$, is much less precise experimentally for two reasons. First, $T_{0}$ is not known precisely enough, and second, $t_{\mathrm{m}}$ is difficult to establish exactly from figure 3 . By way of contrast, $T_{0}$ does not appear in the $\varepsilon_{\mathrm{m}}^{\prime}(E)$ law and $\varepsilon_{\mathrm{m}}^{\prime}$ can be measured much more precisely than $t_{\mathrm{m}}$. Nevertheless, the law $t_{\mathrm{m}}=3 B^{1 / 3} 4^{-2 / 3} A_{0}^{-1} E^{2 / 3}$ using the values of $A_{0}$ and $B^{\prime}$ found above is in approximate agreement with experiment.

The above method is equally useful for calculating the coefficient $C$ (or $B_{6}$ here) of the $P^{6}$ term in the free energy if that term becomes predominant over the $P^{4}$ term. The slope in figure 4 then becomes $4 / 5$ instead of $2 / 3$, as was found experimentally (Bornarel and Troussaut) for $\mathrm{KH}_{2} \mathrm{PO}_{4}$ for applied fields above $4 \mathrm{kV} \mathrm{cm}^{-1}$. For TsCC the $\frac{1}{6} C P^{6}$ term with the value of $C$ found previously (Sorge and Straube 1979) contributes only a few per cent at the right side of figures 4 and 5 , and fields above $80 \mathrm{kV} \mathrm{cm}^{-1}$ would be necessary to make this term predominate!

Values found by various workers for $A_{0}, B$ and $C$ are shown in table 1 . There is a large variation in values of $A_{0}$ whose origin is not clear. The value of $B$ differs from those found by others (Makita 1965, Sorge and Straube 1979), but their values were found from the spontaneous polarisation curve values assuming that $B$ is independent of temperature over a wide temperature range, while our $B$ is determined from measurements near $T_{\mathrm{c}}$. Our method not only has this advantage of determining $B$ (or $C$ ) over a 
narrow temperature range; it also does not require previous or simultaneous determination of $A_{0}$ and $T$, except in determining the adiabatic correction. The fractional adiabatic correction $\left(B^{\prime}-B\right) / B$ is rather small $(0.173)$ for TSCC; for crystals like $\mathrm{KH}_{2} \mathrm{PO}_{4}$ in which it is about 50 times larger (Western and Schmidt 1976), isothermal susceptibility values would be necessary for accurate determinations of $B$ by the method presented in the Appendix.

Table 1. Free-energy parameters $A_{0}, B$ and $C$ for TSCC.

\begin{tabular}{|c|c|c|c|}
\hline Reference & $\begin{array}{l}A_{0} \\
\left(10^{9} \mathrm{~V} \mathrm{~m} \mathrm{~A}^{-1} \mathrm{~s}^{-1} \mathrm{~K}^{-1}\right)\end{array}$ & ${ }^{B}\left(10^{15} \mathrm{~V} \mathrm{~m}^{5} \mathrm{~A}^{-3} \mathrm{~s}^{-3}\right)$ & $\begin{array}{l}C \\
\left(10^{21} \mathrm{Vm}^{9} \mathrm{~A}^{-5} \mathrm{~s}^{-5}\right)\end{array}$ \\
\hline Makita (1965) & $1.9 \pm 0.1$ & $4.6 \pm 0.4$ & \\
\hline Levstik et al (1975) & 6.61 & & \\
\hline Sorge and Straube (1979) & $1.94 \pm 0.04$ & $4.4 \pm 0.1$ & $1.4 \pm 0.2$ \\
\hline Fujimoto et al (1980) & 2.98 & & \\
\hline Schmidt $(1980)$ & $3.65 \pm 0.2$ & & \\
\hline This work & $2.95 \pm 0.1$ & $3.5 \pm 0.1$ & \\
\hline
\end{tabular}

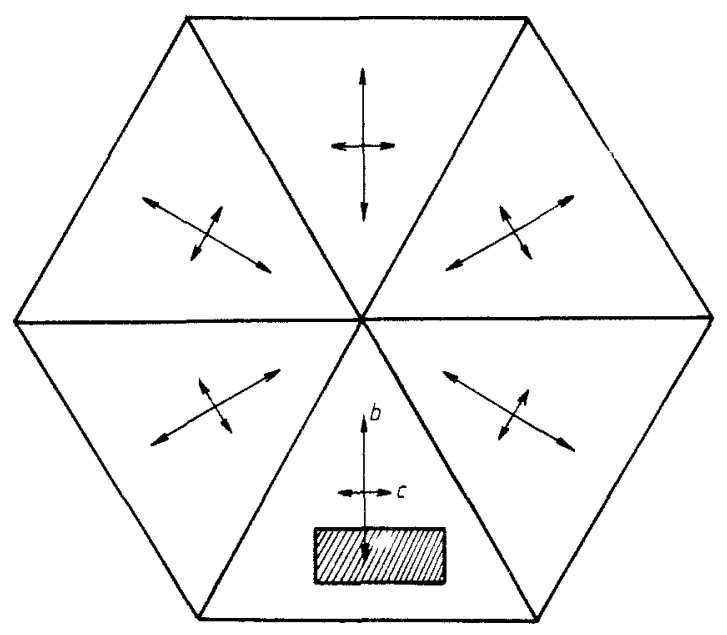

Figure 6. Sketch of projection along $a$ axis of typical ferroelastically polydomain Tscc crystal, showing $b$ axis direction for monodomain portion cleaved from this polycrystal. The asymmetry along $b$ could give rise to defect or impurity gradients which could cause the observed effective internal bias fields.

Several questions remain open after these measurements. First, what is the origin of the internal bias field observed by us and others (Sorge and Straube 1979)? Further studies are desirable with crystals having various concentrations of impurities, taking into account also the relation between the sign of the bias field and the direction of the surface perpendicular to $b$ of the ferroelastic monodomain cleaved from the parent polydomain crystal, as shown in figure 6 . In this connection, optical viewing along the 
$a$ axis during application of DC fields is desirable to ensure that the fields do not create new ferroelastic domains in the originally monodomain samples.

A second question is the origin of the downturn of the $\left(1 / \varepsilon_{\mathrm{m}}^{\prime}\right)^{3 / 2}$ versus $E$ curve in figure 4 at small $1 / \varepsilon_{\mathrm{m}}^{\prime}$. One would expect a rounded minimum resulting from a defectinduced distribution of $T_{\mathrm{c}}$ 's rather than the observed pointed minimum.

Finally, what is the effect of pressure on $B$ ? Does it remain positive up to the three-phase common point at $5.03 \mathrm{kbar}$ (Schmidt 1980), corresponding to the $\mathrm{PE}-\mathrm{FE}$ transition remaining of second order? Similar measurements under hydrostatic pressure are desirable to answer this question.

\section{Acknowledgments}

The authors wish to thank R Mainguenaud and C Villeneuve for very able technical assistance and $\operatorname{Pr}$ Lajzerowicz for fruitful discussions.

\section{Appendix}

We present here a calculation of $\varepsilon_{\mathrm{m}}^{\prime}$ and $t_{\mathrm{m}}$ as functions of $E$, for a Landau free energy of the form

$$
F=\frac{1}{2} A_{0} t P^{2}+\frac{1}{2 n} B_{2 n} P^{2 n}-E P,
$$

where $t=T-T_{0}$, and $\varepsilon_{\mathrm{m}}^{\prime}$ and $t_{\mathrm{m}}$ are for a given field $E$ the maximum real dielectric permittivity and the temperature (less $T_{0}$ ) at which this maximum occurs. By differentiation one obtains the equation of state

$$
E=A_{0} t P+B_{2 n} P^{2 n-1}
$$

which after a second differentiation yields the inverse isothermal susceptibility

$$
\chi_{T}^{-1}=A_{0} t+(2 n-1) B_{2 n} P^{2 n-2} \text {. }
$$

Since we are looking for $\varepsilon_{\mathrm{m}}^{\prime}$ and $t_{\mathrm{m}}$ for curves of fixed DC field $E$, the total derivative of equation (A2) yields

$$
\mathrm{d} P / \mathrm{d} t=-A_{0} P /\left[A_{0} t+(2 n-1) B_{2 n} P^{2 n-2}\right] .
$$

The maximum $\chi_{T}$ occurs where

$$
\mathrm{d} \chi_{T}^{-1} / \mathrm{d} t=A_{0}+(2 n-1)(2 n-2) B_{2 n} P^{2 n-3} \mathrm{~d} P / \mathrm{d} t=0 .
$$

Substitution of equation (A4) into equation (A5) yields

$$
A_{0} t_{\mathrm{m} T}=(2 n-1)(2 n-3) B_{2 n} P_{\mathrm{m}}^{2 n-2}
$$

which together with equations (A2) and (A3) yields

$$
\begin{aligned}
& t_{\mathrm{m} T}=3 B^{1 / 3} E^{2 / 3} / 2^{4 / 3} A_{0}, \\
& \chi_{\mathrm{m} T}^{-1}=3 B^{1 / 3} E^{2 / 3} / 2^{1 / 3},
\end{aligned}
$$

for $n=2$ and $B_{2 n}=B$ as required for our analysis. The approximation = $1+\chi / \varepsilon_{0} \simeq \chi / \varepsilon_{0}$ is sufficiently accurate for this study. Because our measurements were 
made at $400 \mathrm{~Hz}$, we determined the adiabatic susceptibility $\chi_{S}$, which is related to $\chi_{T}$ by

$$
\chi_{\bar{S}}^{-1}=\chi_{T}^{-1}+T C_{P}^{-1}\left[(\delta E / \delta T)_{P}\right]^{2},
$$

(Jona and Shirane 1962), where $C_{P}$ is specific heat per unit volume at fixed $P$. Using equations (A2) and (A3) we obtain

$$
\chi_{S}^{-1}=A_{0} t+\left(3 B+T A_{0}^{2} / C_{P}\right) P^{2}
$$

for the $n=2$ case. From here on we will suppress the subscript $S$. With $\chi_{T}^{-1}$ replaced by this $\chi_{s}^{-1}$ in the foregoing analysis, equation (A6) becomes

$$
A_{0} t_{\mathrm{m}}=3 B P_{\mathrm{m}}^{2}+2 A_{0} T_{0} P_{\mathrm{m}}^{2} /\left(C_{P}-A_{0} P_{\mathrm{m}}^{2}\right),
$$

but the $A P_{\mathrm{m}}^{2}$ term in the denominator can be neglected because $A_{0} P_{\mathrm{m}}^{2} / C_{P}=0.0003$ for the largest field used in this study. Then the analysis proceeds as before, giving

$$
E=4 B\left(1+T_{0} A_{0}^{2} / 2 C_{P} B\right) P_{\mathrm{m}}^{3}=4 B^{\prime} P_{\mathrm{m}}^{3} .
$$

Using the fact that for TSCC a binomial expansion of the factor in parentheses is justified, we find that the equivalents of equations (A7) and (A8) for adiabatic $t_{\mathrm{m}}$ and $\chi_{\mathrm{m}}^{-1}$ have the same form but with $B$ replaced by $B^{\prime}$. The value of $B^{\prime}-B$ is $0.606 \times 10^{15} \mathrm{~V} \mathrm{~m}^{5} \mathrm{~A}^{-3}$ $\mathrm{s}^{-3}$, where $C_{P}$ is calculated using the heat capacity per unit volume of $55 \mathrm{cal} \mathrm{mol}^{-1} \mathrm{~K}^{-1}$ just above $T_{\mathrm{c}}$ (Makita 1965) and the density of $1.53 \mathrm{~g} \mathrm{~cm}^{-3}$ quoted (Ashida et al 1972) for TSCC.

\section{References}

Ashida T, Bando S and Kakudo M 1972 Acta Crystallogr. B28 1560

Fujimoto S, Yasuda N, Takagi K, Narayanan P S and Bhat H L 1980 J. Phys. D: Appl. Phys. 13 L107

Hegenbarth E and Schmidt B 1980 Ferroelectrics 25359

Jona F and Shirane G 1962 Ferroelectric crystals (New York: Pergamon) p 39

Levstik A, Filipič C and Blinc R 1975 Solid State Commun. 181231

Makita Y 1965 J. Phys. Soc. Japan 202073

Sawada A, Makita Y and Takagi Y 1977 J. Phys. Soc. Japan 421918

Schmidt V H 1980 Solid State Commun. 35649

Sorge G and Straube U 1979 Phys. Stat. Solidi (a) 51117

Western A B and Schmidt V H 1976 Solid State Commun. 19885

Windsch W and Schmidt VH 1979 Bull. Am. Phys. Soc. 24507

1980 Ferroelectrics 30 to appear 